\title{
Pain Management in Post-Craniotomy Patients: A Survey of Canadian Neurosurgeons
}

\author{
Basil Hassouneh, John E. Centofanti, Kesava Reddy
}

\begin{abstract}
Introduction: Despite the growing recognition for analgesic needs in post-craniotomy patients, this remains a poorly studied area in neurological surgery. The class and regimen of analgesia that is most suitable for these patients remains controversial. The objective of this study is to examine the current beliefs and practices of Canadian neurosurgeons when managing post-craniotomy pain. Methods: A survey was sent to all practicing Canadian neurosurgeons to examine the following aspects of analgesia in craniotomy patients: type of analgesics used, common side effects encountered, satisfaction with current regimen and the rationale for their practice. Results: Of 156 potential respondents, 103 neurosurgeons (66\%) completed the survey. Codeine (59\%) was the most prescribed firstline analgesic followed by morphine (38\%). The use of a second-line opioid was significantly higher among codeine prescribers compared to morphine, $53 \%$ compared to $28 \%(\mathrm{p}<0.001)$. Nausea, constipation and neurologic depression were reported as common side effects by $76 \%, 66 \%$ and $27 \%$ of respondents respectively. Of the respondents, $90 \%$ reported a high level of satisfaction with their current choice of analgesia; nonetheless, they predominantly described their practice as personal preference or protocol driven rather than evidence-based. Conclusions: Codeine - a weak opioid - is the most common first-line analgesic prescribed to craniotomy patients. This practice is associated with substantially increased reliance on potent opioids for rescue analgesia. Whether novel regimens can provide optimal pain control while minimizing neurologic and gastrointestinal side effects remains to be addressed by future trials.
\end{abstract}

RÉSUMÉ: Enquête auprès des neurochirurgiens canadiens sur le traitement de la douleur chez les patients qui ont subi une craniotomie. Contexte : Même si la nécessité d'administrer des analgésiques chez les patients qui ont subi une craniotomie est maintenant mieux établie, c'est un sujet qui a été peu étudié. La classe et le régime posologique d'analgésiques qui convient le mieux à ces patients demeurent controversés. 'objectif de cette étude était d'examiner les convictions et les pratiques actuelles des neurochirurgiens canadiens concernant le soulagement de la douleur après une craniotomie. Méthode : Une enquête a été faite auprès de tous les neurochirurgiens canadiens actuellement en pratique pour examiner les aspects suivants concernant l'analgésie chez les patients qui ont subi une craniotomie : le type d'analgésiques utilisés, les effets secondaires fréquemment observés, le degré de satisfaction concernant les régimes posologiques actuels et la justification de leurs habitudes de prescription. Résultats : Des 156 répondants potentiels, 103 neurochirurgiens (66\%) ont complété l'enquête. L'analgésique de première ligne le plus fréquemment prescrit était la codéine (59\%), suivi de la morphine (38\%). L'utilisation d'un opiö̈de de deuxième ligne était significativement plus élevée parmi les prescripteurs de codéine que parmi les prescripteurs de morphine, soit $53 \%$ par rapport à $28 \%(\mathrm{p}<0,001)$. Les effets secondaires les plus courants étaient les nausées, la constipation et la dépression neurologique, rapportés par $76 \%, 66 \%$ et $27 \%$ des répondants respectivement. De plus, $90 \%$ d'entre eux ont rapporté un haut degré de satisfaction concernant leur choix actuel d'analgésique. Cependant, ils ont décrit leur choix comme étant motivé par une préférence personnelle ou un protocole plutôt que fondé sur des preuves. Conclusions : La codéine, un opioïde faible, est l'analgésique de première ligne le plus fréquemment prescrit chez les patients qui ont subi une craniotomie. Cette pratique est associée à un recours à des opioïdes puissants comme analgésique de rattrapage. Il faudra effectuer des essais cliniques pour évaluer si de nouveaux régimes de traitement pourraient optimiser le contrôle de la douleur tout en minimisant les effets secondaires neurologiques et gastro-intestinaux.

Can. J. Neurol. Sci. 2011; 38: 456-460

Craniotomy is a common operative procedure in neurosurgical practice. It involves opening a window in the bony cranium to allow surgical access to the intra-cranial content. The brain is believed to lack pain sensation, and most such innervation is limited to the meningeal covering or the extracranial muscle and fascia. ${ }^{1}$ This concept of insensate brain has led to a widespread perception that craniotomy patients experience minimum post-operative pain, and therefore their analgesia received little attention in the past. Subsequently, several reports have challenged this belief and suggested that patients experience moderate to severe pain following craniotomy. ${ }^{2-4}$ Post-operative pain appears to be particularly intense when extensive muscle dissection or infra-tentorial approaches are used.
Although it is becoming clear that adequate pain control is an important component of post-craniotomy care, opinions have varied regarding the most suitable analgesia regimen. ${ }^{5-9}$ Postsurgical pain is often moderate to severe in the immediate postoperative period. Potent opioids (narcotics), such as morphine,

From the Department of Clinical Epidemiology \& Biostatistics (BH), Department of Anesthesia (JEC), Department of Surgery - Division of Neurosurgery (KR), McMaster University, Hamilton, Ontario, Canada.

Received October 4, 2010. Final Revisions Submitted December 1, 2010 Correspondence to: Basil Hassouneh, Health Research Methodology Program, McMaster University, HSC-2C1 - 1200 Main St. W., Hamilton, Ontario, L8N 3Z5, Canada. 
are the cornerstone analgesics to adequately manage this pain. ${ }^{10}$ Codeine is viewed as a weak opioid and is less suited to control moderate to severe pain as a single agent. Furthermore, it is ineffective in up to $15 \%$ of the population due to deficiencies in the enzymes needed to convert codeine into its active metabolites. ${ }^{11}$ Despite the previous popularity of codeine in neurosurgical units, several studies favoured using potent opioids as a safe and more effective alternative. ${ }^{12-14}$ Recently, small trials have explored the use of patient-controlled fentanyl analgesia as well as tramadol with promising initial results. ${ }^{15}, 16$ Nonetheless, potent opioids have potential disadvantages: oversedation can interfere with the critical neurologic monitoring, and suppressed ventilation can lead to hypercapnia that may increase cerebral blood flow and exacerbate brain edema. ${ }^{17-19}$

Acetaminophen is commonly used as an ancillary analgesic to lower opioid requirements; however, it remains insufficient as a single agent to control post-operative pain. Novel non-steroidal anti-inflammatory drugs (NSAIDs) and cyclooxygenase-2 (COX-2) inhibitors are emerging as attractive adjuncts to traditional analgesics. ${ }^{20-22}$ A small study by Rahimi et al suggested that combining opioids and COX-2 inhibitors can achieve adequate pain control while reducing the undesirable effects of opioids in post-craniotomy patients. ${ }^{20}$ Nonetheless, the anti-platelet effects of NSAIDs as well as the potential risk for cardiovascular events are still significant drawbacks to many neurosurgeons. ${ }^{22,23}$ Adequate randomized trials to contrast different analgesia regimens are unavailable in the neurosurgical population. There is limited evidence to support a specific class or regimen in providing optimal pain control while minimizing side effects and risks. Understanding the perceptions and attitudes towards analgesia is an important step to inform and guide future trials. Therefore, the objective of this study is to examine the current beliefs and practices of Canadian neurosurgeons when managing post-craniotomy pain.

\section{Methods and Survey Design}

Currently practicing neurosurgeons in Canada were identified using listings from the following resources: Canadian Clinical

- What is the first-line analgesic given to post-craniotomy patients in the immediate postoperative period?

- Why do you use this drug?

- Do you use another drug for breakthrough?

- In your experience, what are the common side effects associated with this pain control regimen?

- What do you find works well to counter the side effects?

- How happy are you with the current pain control in your post-craniotomy patients?

- Any comments or suggestions?

Figure 1: Summary of the administered survey. Both choice-list and open-end responses are used to allow for flexibility in the reporting.
Neurosciences Society, College of Physicians and Surgeons for each province and academic neurosurgery departments. Retired neurosurgeons or residents in-training were excluded. Surveys were mailed, emailed or faxed to a total of 157 identified candidates in their primary language of correspondence (English or French). Non-respondents were sent reminders. The survey was composed of five focused questions examining the following aspects of analgesia in elective post-craniotomy patients: analgesics used, common side effects encountered, satisfaction with current regimen and rationale for the practice. Both choice-list and open-ended responses were used in the survey to allow for flexibility in the reporting. Participants also had the opportunity to provide comments and suggestions regarding analgesia in their patient population. The survey was pre-tested and edited to resolve potential sources of confusion and ensure clear comprehension of the examined items. Figure 1 provides a summary of the administered survey.

\section{Statistical Analysis}

The statistical analysis and testing was performed using SPSS 15 statistical software. Descriptive statistics were presented as percentages of total respondents. Ordinal data was collapsed into abridged ordinal or dichotomous variables to allow statistical testing. Spearman's correlation coefficient, Pearson's chi-square test and Fisher's exact test were used as suitable.

\section{First-line Analgesia}

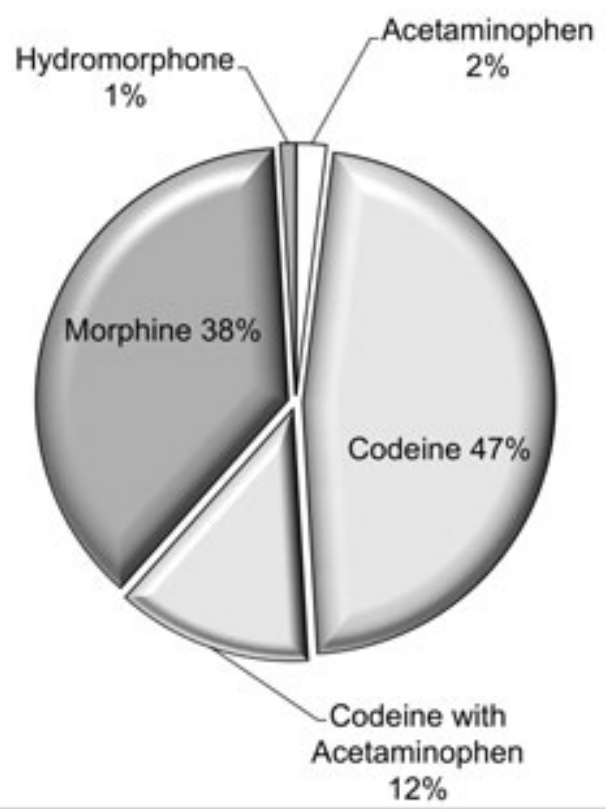

Figure 2: First-line analgesics reported by neurosurgeons. 


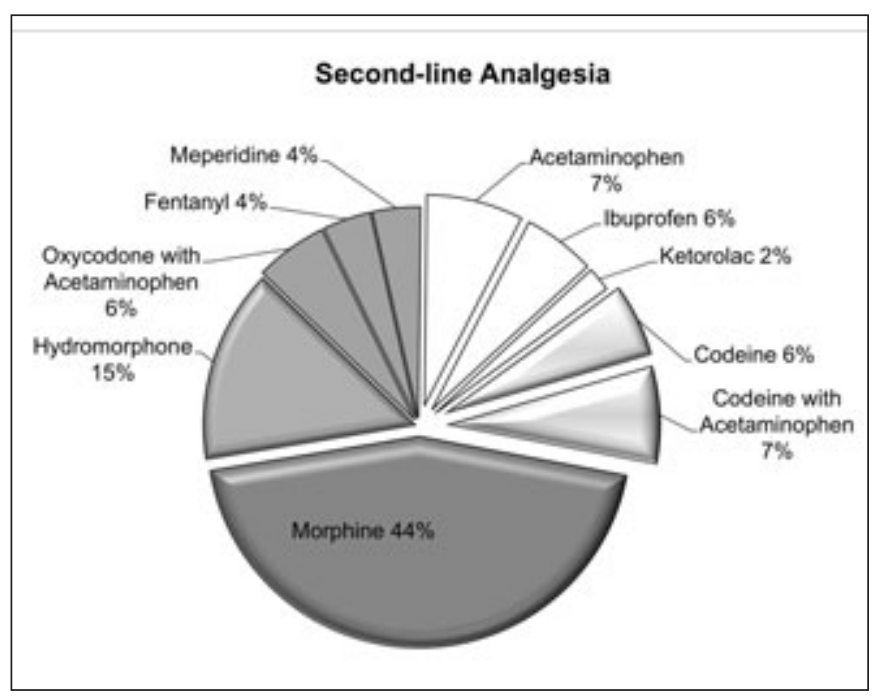

Figure 3: Second-line analgesics reported by neurosurgeons.

\section{RESULTS}

The survey achieved an overall participation rate of $66 \%$ from practicing Canadian neurosurgeons. The province of Quebec had a markedly lower response rate, $20 \%$, compared to the rest of Canada, $85 \%$. Figure 2 presents the reported first-line analgesics. The most prescribed first-line was codeine followed by morphine, $59 \%$ and $38 \%$ of respondents respectively. Codeine and morphine were most frequently administered as 30-60 mg oral/intramuscular q 3-4 hr, and 2-4 mg intravenous q 2-3 hr, respectively. The use of second-line analgesics was reported by

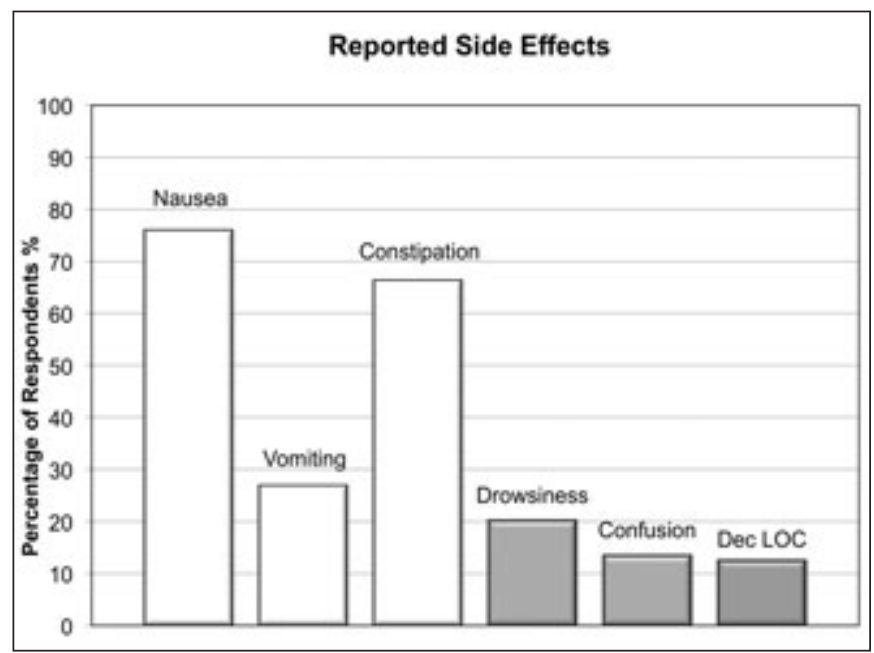

Figure 4: The common side effects of post-craniotomy analgesia. The $x$ axis shows the percentage of neurosurgeons reporting each side effect. Gastrointestinal side effects include nausea, vomiting and constipation. Neurologic depression includes drowsiness, confusion and decreased level of consciousness (Dec LOC).
$52 \%$ of neurosurgeons. Figure 3 presents the spectrum of second-line choices. Morphine was by far the most prescribed second-line (44\%); it was predominantly used in conjunction with codeine as the first-line agent. The use of a potent secondline opioid was significantly higher among first-line codeine prescribers compared to morphine, $53 \%$ compared to $28 \%$ (p < $0.001)$.

Figure 4 presents the common side effects encountered. Gastrointestinal complaints were the most dominant with nausea and constipation reported by $76 \%$ and $66 \%$ of respondents, respectively. Symptoms of neurologic depression including confusion, drowsiness or decreased level of consciousness were recognized as common side effects by $27 \%$ of respondents. When asked about satisfaction, the majority of neurosurgeons displayed a high level of satisfaction with their current choice of

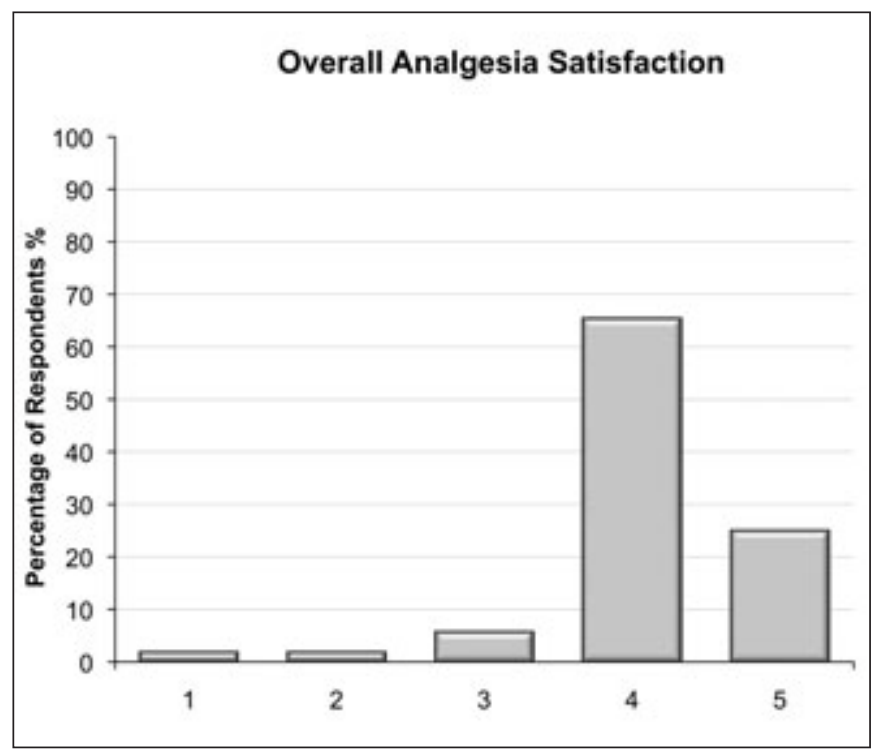

Figure 5: Neurosurgeons' level of satisfaction with their analgesia regimen. The $x$-axis shows the percentage of neurosurgeons reporting each score. 1: very unsatisfied, 2: unsatisfied, 3: neither unsatisfied nor satisfied, 4: satisfied, 5: very satisfied.

analgesia regimen. Figure 5 details the reported satisfaction scores: $90 \%$ were very satisfied or satisfied (scores 5 and 4) with the current pain control; $10 \%$ were slightly satisfied or unsatisfied (scores 3,2 and 1). The neurosurgeons overwhelmingly reported that their choice of analgesia was based on personal preferences or established postoperative protocols. Only 5\% thought that their choice was supported by clinical evidence. Several factors were examined for possible correlation with the frequency of side effects or the reported level of satisfaction: there was no statistically significant difference with regard to the choice of opioid (codeine vs. morphine), maximum prescribed dose/day (high versus low) or use of second-line analgesia (used versus not used). 


\section{DISCUSSION AND CONCLUSIONS}

The results indicate that codeine - a weak opioid - continues to be the dominant choice, accounting for the majority of the prescribed first-line analgesics. The use of a potent second-line opioid was substantially higher in first-line codeine prescribers compared to those who chose morphine as the initial agent. This may reflect an inadequate pain control with codeine as the primary analgesic. These findings share some similarity with previous reports from British neurosurgical units suggesting a widespread analgesia under-prescription., ${ }^{7,24}$ Roberts et al reported that $80 \%$ of British neurosurgeons utilized codeine phosphate as a first-line analgesic, compared to only $8 \%$ using morphine. ${ }^{24}$ Codeine is commonly viewed as an inactive agent that requires bioconversion into active metabolites predominantly morphine. Cytochrome P-450 2D6 (CYP2D6) plays a critical role in codeine metabolism. ${ }^{11,25,26}$ There is wide variability amongst races and individuals in the activity of CYP2D6 due to high genetic polymorphism and functional allele duplication. This can explain why "poor metabolizers" show no analgesic response to codeine, whereas at the other extreme end, "ultra-rapid metabolizers" exhibit signs of codeine toxicity even with presumably normal dosages. Several novel opioids are becoming accepted alternatives in post-operative pain management today. Tramadol, a synthetic opioid, has demonstrated good efficacy and safety in surgical patients, and a recent small trial showed promising results in post-craniotomy patients. ${ }^{16}$ Hydromorphone and oxycodone are other potent semi-synthetic opioids in current use. Although hydromophone has been well studied in the management of acute pain, some of its metabolites are associated with neuroexcitatory states which is concerning in neurosurgical patients. ${ }^{27,28}$ Oxycodone has demonstrated reliable efficacy and a favourable side effect profile in several trials; furthermore, its high bioavailability compared to morphine makes it advantageous when transitioning from parenteral to oral administration. ${ }^{29-31}$ These novel agents remain largely underutilized in neurosurgical units with only a limited second-line role reported in this survey.

The careful assessment of potential side effects and their impact on craniotomy patients is critical when choosing an analgesia regimen. Opioid effects on the neurologic status are particularly important in this sensitive population. Signs of neurologic depression such as confusion, drowsiness or decreased level of consciousness were recognized as "common" side effects by a remarkable $27 \%$ of respondents. These symptoms can interfere with the neurologic monitoring or even misleadingly suggest an intracranial event. Gastrointestinal side effects were frequently reported, and many respondents indicated using anti-emetics or laxatives routinely to minimize these symptoms. Nausea and vomiting can be particularly problematic as this can elevate intracranial pressure or increase the risk for aspiration if the laryngeal reflexes are blunted. The traditional perception in the past was that sedation, respiratory suppression and gastrointestinal side effects were less with codeine compared to its counterpart morphine. ${ }^{17}$ Nonetheless, the available randomized trials are inconsistent, and some studies suggests that the presumed "lower" side effect profile is lost when dosing is adjusted to account for the significant variation in codeine metabolism. ${ }^{11,12,14}$
Non-opioids such as NSAIDs, COX-2 inhibitors and acetaminophen are important classes in the analgesia armamentarium. In this survey, neurosurgeons reported using non-opioids mostly as second-line or adjunct analgesics. The use of NSAIDs was limited to a small second-line role, $8 \%$ comprised of ibuprofen and ketorolac, whereas COX-2 inhibitors were not utilized. Although these classes can avoid neurologic depression, they raise concerns due to the potential risk for intracranial bleeding or cardiovascular events. ${ }^{22,23,32}$ The limited use of these agents may reflect hesitation to accept such risks along with unchallenged satisfaction with the current opioid analgesia. Acetaminophen was predominantly used in conjunction with codeine, often as Tylenol with codeine preparations. Its limited role as a single agent is in keeping with a mild analgesic effect.

The reported level of satisfaction with the analgesia regimen was rather high and unrelated to the choice of opioid or the dose prescribed. Surgeon impressions may, however, differ from what is found in patient-based clinical studies. Indeed, the high level of neurosurgeon satisfaction exhibited here contrasts with reports showing a lower patient satisfaction, with significant experience of moderate to severe pain following craniotomy. . $^{2,3,14}$ Despite the growing recognition for analgesic needs in craniotomy patients, this remains a poorly studied area of neurological surgery. In this survey, neurosurgeons overwhelmingly agreed that their current practices are not guided by clinical evidence, but rather reflect personal preferences or established protocols.

This survey examined important aspects of the attitudes and practices adopted by Canadian neurosurgeons when managing pain in elective craniotomy patients. The items focused on the type of analgesics used, side effects encountered and satisfaction with the treatment. This approach achieved a high response rate with the exception of Quebec. The latter is likely due to our restricted ability to access updated contact information for this province. It is important to recognize some of the limitations encountered with survey designs. Analgesia regimens are often prescribed with a range of dose, frequency and threshold for clinical administration. Accordingly, this survey cannot be used to determine the exact analgesics administered or to isolate their effects, but rather to evaluate the general practices adopted by neurosurgeons. The results obtained from clinical studies may differ from what neurosurgeons have reported. This is particularly important when considering pain control or side effects and their impact on patients since physician-based perception can be unreliable. On the other hand, the reported analgesia regimens are unlikely to differ significantly from the actual clinical practice since these were mostly driven by established preferences or protocols.

In conclusion, codeine remains the most widely used firstline analgesic in Canadian neurosurgical units despite its both weak and variable analgesic properties. This choice is strongly associated with increased reliance on potent rescue analgesia. The current practice is likely driven by multiple factors including: fear of opioid sedation, under recognition of postcraniotomy pain, and established analgesia preferences or protocols. We hope that these findings will encourage neurosurgeons to re-examine their analgesia practices, and pursue collaboration on definitive trials that challenge the 
current codeine predominance against potentially more effective and favourable analgesia regimens. A specific concern is the risk of opioid sedation that may interfere with the post-operative neurologic assessment leading to unnecessary computed tomogram scans, prolonged stay in high-observation units and delayed rehabilitation. Such outcomes need to be assessed in any opioid trial to adequately address the traditional concern of excessive sedation. Whether NSAIDs, COX-2 inhibitors, novel opioids or mixed regimens can provide optimal pain control while minimizing neurologic and gastrointestinal side effects remains to be addressed by future trials.

\section{NoTE}

B. Hassouneh and J. E. Centofanti contributed equally to this study.

\section{ACKNowledgement}

The authors thank Dr. Gordon Guyatt for providing advice on the manuscript draft.

\section{REFERENCES}

1. Dunbar PJ, Visco E, Lam AM. Craniotomy procedures are associated with less analgesic requirements than other surgical procedures. Anesth Analg. 1999;88(2):335-40.

2. Gottschalk A, Berkow LC, Stevens RD, et al. Prospective evaluation of pain and analgesic use following major elective intracranial surgery. J Neurosurg. 2007;106(2): 210-16.

3. Thibault M, Girard F, Moumdjian R, et al. Craniotomy site influences postoperative pain following neurosurgical procedures: a retrospective study. Can J Anaesth. 2007;54(7): 544-8.

4. Ortiz-Cardona J, Bendo AA. Perioperative pain management in the neurosurgical patient. Anesthesiol Clin. 2007;25(3):655-74.

5. Talke PO, Gelb AW. Postcraniotomy pain remains a real headache! Eur J Anaesthesiol. 2005;22(5):325-7.

6. Durieux ME, Himmelseher S, Durieux ME, Himmelseher S. Pain control after craniotomy: off balance on the tightrope? J Neurosurg. 2007;106(2):207-9.

7. Kotak D, Cheserem B, Solth A. A survey of post-craniotomy analgesia in British neurosurgical centres: time for perceptions and prescribing to change? Br J Neurosurg. 2009;23(5):538-42.

8. Gottschalk A. Craniotomy pain: trying to do better. Anesth Analg. 2009;109(5):1379-81

9. Jutsum FJ. Time for perceptions and prescribing to change? Br J Neurosurg. 2010;24(1):94.

10. Nemergut EC, Durieux ME, Missaghi NB, et al. Pain management after craniotomy. Best Pract Res Clin Anaesthesiol. 2007;21(4): $557-73$.

11. Poulsen L, Brosen K, Arendt-Nielsen L, Gram LF, Elbaek K, Sindrup SH. Codeine and morphine in extensive and poor metabolizers of sparteine: pharmacokinetics, analgesic effect and side effects. Eur J Clin Pharmacol. 1996;51(3-4):289-95.

12. Goldsack C, Scuplak SM, Smith M. A double-blind comparison of codeine and morphine for postoperative analgesia following intracranial surgery. Anaesthesia. 1996;51(11):1029-32.

13. Stoneham MD, Cooper R, Quiney NF, Walters FJ. Pain following craniotomy: a preliminary study comparing PCA morphine with intramuscular codeine phosphate. Anaesthesia. 1996;51(12): $1176-8$.
14. Sudheer PS, Logan SW, Terblanche C, Ateleanu B, Hall JE. Comparison of the analgesic efficacy and respiratory effects of morphine, tramadol and codeine after craniotomy. Anaesthesia. 2007;62(6):555-60.

15. Morad AH, Winters BD, Yaster M, et al. Efficacy of intravenous patient-controlled analgesia after supratentorial intracranial surgery: a prospective randomized controlled trial. Clinical article. J Neurosurg. 2009;111(2):343-50.

16. Rahimi SY, Alleyne CH, Vernier E, Witcher MR, Vender JR. Postoperative pain management with tramadol after craniotomy: evaluation and cost analysis. J Neurosurg. 2010;112(2):268-72.

17. Stoneham MD, Walters FJ. Post-operative analgesia for craniotomy patients: current attitudes among neuroanaesthetists. Eur J Anaesthesiol. 1995;12(6):571-5.

18. Roberts G. A review of the efficacy and safety of opioid analgesics post-craniotomy. Nurs Crit Care. 2004;9(6):277-83.

19. Cold GE, Felding M. Even small doses of morphine might provoke "luxury perfusion" in the postoperative period after craniotomy. Neurosurgery. 1993;32(2):327.

20. Rahimi SY, Vender JR, Macomson SD, et al. Postoperative pain management after craniotomy: evaluation and cost analysis. Neurosurgery. 2006;59(4):852-7; discussion 857.

21. Jones SJ, Cormack J, Murphy MA, Scott DA. Parecoxib for analgesia after craniotomy. Br J Anaesth. 2009;102(1):76-9.

22. Umamaheswara Rao GS, Gelb AW. To use or not to use: the dilemma of NSAIDs and craniotomy. Eur J Anaesthesiol. 2009; 26(8):625-6.

23. Palmer JD, Sparrow OC, Iannotti F. Postoperative hematoma: a 5year survey and identification of avoidable risk factors. Neurosurgery. 1994;35(6):1061-4; discussion 1064-5.

24. Roberts GC. Post-craniotomy analgesia: current practices in British neurosurgical centres--a survey of post-craniotomy analgesic practices. Eur J Anaesthesiol. 2005;22(5):328-32.

25. Wilkinson GR. Drug metabolism and variability among patients in drug response. N Engl J Med. 2005;352(21):2211-21.

26. Kirchheiner J, Schmidt H, Tzvetkov M, et al. Pharmacokinetics of codeine and its metabolite morphine in ultra-rapid metabolizers due to CYP2D6 duplication. Pharmacogenomics J. 2007;7(4): 257-65.

27. Quigley C, Wiffen P. A systematic review of hydromorphone in acute and chronic pain. J Pain Symptom Manage. 2003;25(2): $169-78$.

28. Murray A, Hagen NA. Hydromorphone. J Pain Symptom Manage. 2005;29 Suppl 5:S57-66.

29. Riley J, Eisenberg E, Muller-Schwefe G, Drewes AM, ArendtNielsen L. Oxycodone: a review of its use in the management of pain. Curr Med Res Opin. 2008;24(1):175-92.

30. Tanskanen P, Kytta J, Randell T. Patient-controlled analgesia with oxycodone in the treatment of postcraniotomy pain. Acta Anaesthesiol Scand. 1999;43(1):42-5.

31. Lenz H, Sandvik L, Qvigstad E, Bjerkelund CE, Raeder J. A comparison of intravenous oxycodone and intravenous morphine in patient-controlled postoperative analgesia after laparoscopic hysterectomy. Anesth Analg. 2009;109(4):1279-83.

32. Kearney PM, Baigent C, Godwin J, Halls H, Emberson JR, Patrono C. Do selective cyclo-oxygenase-2 inhibitors and traditional non-steroidal anti-inflammatory drugs increase the risk of atherothrombosis? Meta-analysis of randomised trials. BMJ. 2006;332(7553):1302-8 